\title{
La curva de aprendizaje y su contribución al desempeño del talento humano en las organizaciones: una revisión teórica
}

\author{
The learning curve and its contribution to the human talent performance in \\ organizations: a theoretical review
}

\author{
Diego Alonso Cardona-Arbeláez ${ }^{1}$ \\ Jorge Luis Del Río-Cortina² \\ Ana Karina Romero-Severiche ${ }^{3}$ \\ Harold Lora-Guzmán ${ }^{4}$
}

Recibido: octubre 10 de 2018

Aceptado: abril 09 de 2019

\section{Resumen}

Este documento presenta una revisión sobre el concepto de curva de aprendizaje, sus orígenes, modelos y principales aplicaciones. Se realizó una investigación documental, para la cual se consultaron bases de datos especializadas, abordando los principales autores y los aspectos característicos de cada modelo. Los hallazgos indican la importancia del estudio de las curvas de aprendizaje, tanto a nivel individual, grupal y organizacional. Estas últimas pueden ser fuente para la toma de decisión en las empresas, ya que los distintos modelos de curva de aprendizaje pueden brindar información, referente a: metas de producción, evaluación de rendimientos, estimación de mano de obra, optimización de precios y costos, para crear ventajas competitivas. Los trabajos revisados destacan el beneficio de las curvas de aprendizaje en temas como: la planeación, la capacitación y el control de procesos. Se concluye que las curvas de

\begin{abstract}
The aim of this article is to carry out a theoretical review of the learning curve, its origins, models and main applications. A documentary investigation was carried out, for which specialized databases were consulted, addressing the main authors and the characteristic aspects of each model. The findings indicate the importance of studying learning curves, both at the individual, group and organizational levels. The latter can be a source for decision-making in companies, since the different learning curve models can provide information referring to: production goals, performance evaluation, labor estimation, and price and cost optimization, to create competitive advantages. The reviewed works highlight the benefit of learning curves in topics such as: planning, training and process control. It is concluded that the learning curves, although they began in the aviation industry, have been disseminated to various industrial
\end{abstract}

1 Administrador de Empresas, Estudiante de Doctorado en Administración, Universidad Libre, Cartagena, Colombia. E-mail: diego. cardona@unilibrectg.edu.co ORCID: 0000-0002-9123-0156

2 Administrador de Empresas, Estudiante de Doctorado en Administración, Universidad Tecnológica de Bolívar, Cartagena, Colombia. E-mail: jdelrio@utb.edu.co ORCID: 0000-0001-6127-8450

3 Economista, Magíster en Gestión y Evaluación de Proyectos de Inversión, Universidad Pontificia Bolivariana, Montería, Colombia. E-mail: anakseveriche@gmail.com ORCID: 0000-0003-2998-0754

4 Ingeniero de Productividad y Calidad, Doctor en Cultura y Educación, Universidad de Cartagena, Cartagena, Colombia. E-mail: hlorag@unicartagena.edu.co ORCID: 0000-0001-6945-0999 
aprendizaje, si bien iniciaron en la industria de la aviación, se han difundido a diversos sectores industriales, con la aportación de nuevos modelos matemáticos para su estimación.

Palabras clave: organización, curva de aprendizaje, conocimiento, desempeño, transferencia de conocimiento.

\section{Introducción}

Desde el momento que el aprendizaje organizacional fue relacionado con la gestión en las organizaciones (Cangelosi \& Dill, 1965), se ha considerado que en la medida en que los individuos aprenden nuevos procedimientos o mejoran las técnicas que utilizan, pueden obtener mejores resultados en sus labores y en las empresas donde se desempeñan. Esto se debe a que el aprendizaje organizacional aumenta el rendimiento de la empresa, a medida que aumenta el tiempo (Ulrich, Von Glinow, Jick, Yeung \& Nason, 1994).

El aprendizaje organizacional debe considerarse como una serie de interacciones entre la adaptación a nivel individual, o subgrupo, y la adaptación a nivel organizacional (Cangelosi \& Dill, 1965). De esta forma, una organización aprende, si una de sus unidades adquiere conocimientos que serán potencialmente útiles para la organización (Huber, 1991).

Por otra parte, Argyris y Schon (1978), consideran que las organizaciones son similares a las personas y depende de ellas el construir aprendizaje colectivo. Igualmente, Huber (1991), expone que el aprendizaje está basado en las personas y se forma de manera gradual. No obstante, Simon (1991), plantea que el aprendizaje sólo se da de manera individual, mientras que la organización aprende de dos maneras: por el aprendizaje de sus miembros y por la inclusión de nuevos miembros, que tienen un conocimiento diferente a los que existen en la sectors, with the contribution of new mathematical models for their estimation.

Keywords: organization, learning curve, knowledge, performance, knowledge transfer.

organización. A su vez, Fiol y Lyles (1985), se refieren a la existencia de acuerdos que deben hacerse para distinguir entre aprendizaje individual y organizacional. Ellos indican que el aprendizaje individual es importante para las organizaciones; sin embargo, el aprendizaje organizacional no es simplemente la suma del aprendizaje de cada miembro.

El aprendizaje organizacional, para Argyris y Schon (1978), se desarrolla a través de actores particulares cuyas acciones se basan en un conjunto de modelo compartido. Igualmente, Kim (1993), relaciona el aprendizaje organizacional con el aprendizaje individual, considerando que una organización aprende a través de sus miembros; por lo tanto, es afectada directa e indirectamente por el aprendizaje individual. En contraste, Cook y Yanow (1993), plantean que las organizaciones aprenden de manera colectiva y no individual. Además, Hult, Ketchen y Reus (2001), indican que el aprendizaje organizacional es la capacidad de desarrollar conocimiento dentro de la organización.

Por su parte, Levitt y March (1988), interpretaron la construcción de aprendizaje organizacional a través de las rutinas en las que se basa la organización, historias dependientes y el hecho de que las organizaciones están orientadas a objetivos. Posteriormente, Huber (1991), expuso cuatro constructos integralmente vinculados al proceso de aprendizaje, con sus respectivos subconstructos, que son: adquisición de conocimiento, distribución de la información, interpretación de la información y memoria organizacional. 
(...) La adquisición de conocimiento, es el proceso por el cual se obtiene el conocimiento. La distribución de la información, es el proceso donde se comparte la información de diferentes fuentes y con ello se conduce a una nueva información o entendimiento. La interpretación de la información, es el proceso por el cual la distribución de la información se da a uno o más, comúnmente atendiendo las interpretaciones. La memoria organizacional, es el medio por el cual el conocimiento se almacena para el uso futuro. (Huber, 1991, p. 90)

Por otra parte, Crossan et al. (1999), difiere de Levitt y March (1988), y de Huber (1991), considerando que el aprendizaje organizacional se desarrolla en cuatro procesos: intuición, interpretación, integración e institucionalización. En contraste, Sinkula (1994) coincide con Huber (1991) en relacionar el aprendizaje organizacional con un proceso compuesto por múltiples aspectos, como: adquisición de información, difusión de información y aplicación conjunta de la información.

En este sentido, Del Río, Cardona y Guacarí (2017), Consideran que el aprendizaje organizacional es el proceso a través del cual las entidades generan y obtienen conocimiento de los trabajadores, con el fin último de integrarlo al desarrollo de sus actividades, logrando así un cúmulo de conocimiento para afrontar entornos cambiantes. Cabe indicar que la construcción de conocimiento reviste complejidad en todos los niveles de la organización, pues el conocimiento organizacional va más allá del simple conocimiento aprendido, ya que este se genera a partir de la relación individuo-organización (Alean et al., 2017).

A partir de las divisiones de procesos o constructos que intervienen en el aprendizaje organizacional, expuestas por Huber (1991) y Levitt y March (1988), se puede resaltar que ambos incluyen Learning by Doing, como una de las características referentes a la adquisición de conocimiento. Siendo de gran importancia para este estudio profundizar en la literatura referente a aprendizaje experimental, se toma como referencia la perspectiva expuesta por Huber (1991), específicamente el subproceso basado en la experiencia, denominado "Curvas de aprendizaje". El objetivo de este artículo es realizar una revisión bibliográfica donde se desarrolle una integración conceptual de las curvas de aprendizaje (ver figura 1), desde la perspectiva Learning by Doing.

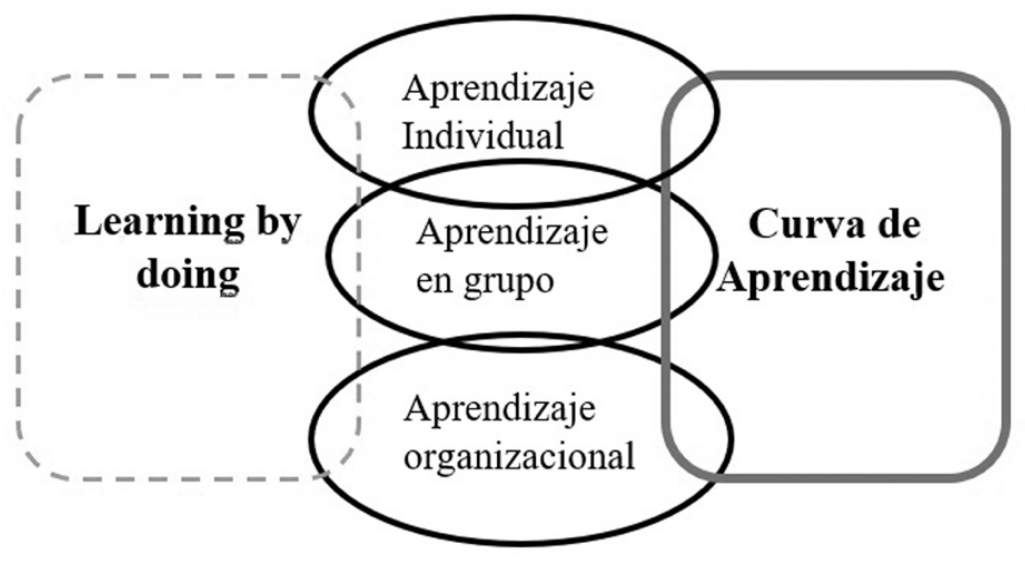

Figura 1. Integración conceptual de las curvas de aprendizaje. 
En las siguientes secciones se exponen: el concepto Learning by Doing y su relación con las curvas de aprendizaje, una revisión de los principales autores que desarrollan el concepto de curva de aprendizaje, los tipos y modelos de curvas de aprendizaje, las críticas realizadas a los modeloss, junto con una explicación de las extensiones de los modelos iniciales; y por último se presentan las conclusiones.

\section{Aprendizaje experimental}

El aprendizaje es producto de la experiencia, considerando que este tiene lugar a través del intento de resolver un problema (Fernández \& González, 2017). En este sentido, sólo se desarrolla durante la actividad y es a través de las experiencias anteriores que se modifica la percepción del individuo (Ordóñez, Gualdrón \& Amaya, 2019). Sin embargo, el aprendizaje experimental asociado con la repetición del mismo problema, disminuye drásticamente los rendimientos. Por consiguiente, tener un mayor rendimiento, implica que las situaciones de estímulo deben a sí mismo estar evolucionando constantemente en lugar de simplemente repetir (Arrow, 1962).
Las empresas, después de su nacimiento, adquieren parte de sus conocimientos a través de la experiencia directa; en algunas ocasiones este aprendizaje es el resultado de esfuerzos deliberados y sistemáticos. Sin embargo, con mayor frecuencia se adquiere involuntariamente, es decir, no sistemático (Huber, 1991). Así mismo, aprender de la experiencia directa, desde la perspectiva de las rutinas y creencias, se da por dos mecanismos. El primero es ensayo y error; y el segundo es la búsqueda de la organización de rutinas alternativas y su adopción cuando son mejores a las que se usan normalmente (Levitt \& March, 1988).

El proceso de aprendizaje experimental, según Huber (1991), es considerado un subconstructo de la adquisición de conocimiento, y este a su vez se divide en subconstructos. En la figura 2, se observan en detalle la clasificación expuesta por el autor, siendo el subconstructo curvas de aprendizaje, el que se analizará con mayor profundidad en los siguientes apartados.

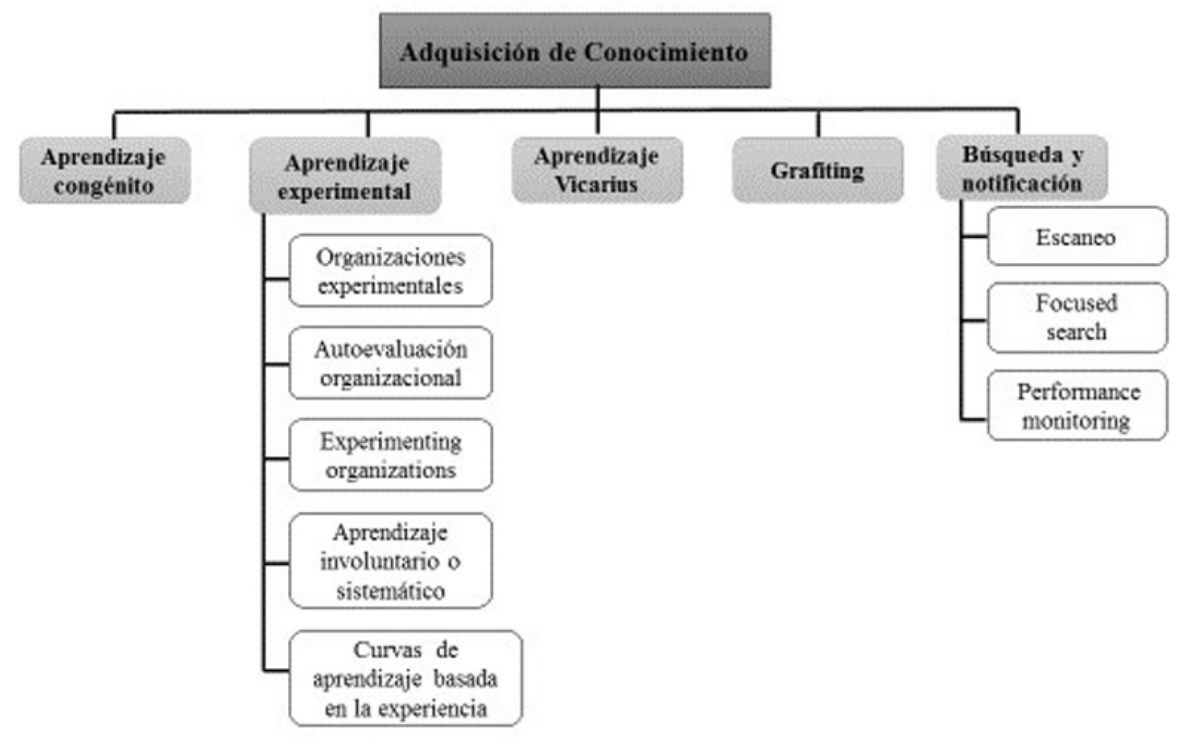

Figura 2. Curva de aprendizaje como un subproceso del aprendizaje experimental. 


\subsection{Curvas de aprendizaje}

El aprendizaje desde la experiencia, se encuentra en los efectos de la producción acumulada y el uso de la experiencia en la manufactura, con el fin de mejorar la productividad (Dutton, Thomas \& Butles, 1984). La experiencia genera un efecto positivo en el desempeño organizacional; es decir, una empresa manufacturera gana experiencia produciendo un nuevo producto, su costo de producción y tiempo por unidad decrecen en la medida que se acumule experiencia en la producción de este. La magnitud de reducción se puede predecir, a partir de un modelo matemático llamado curva de experiencia o curva de aprendizaje (Huber, 1991).

Sin embargo, Hall y Howell (1985), realizan una distinción entre los términos curva de experiencia y curva de aprendizaje". Esta última se refiere a los costos de mano de obra por sí solos, y la aplicación del aprendizaje mediante la práctica a corto plazo. En contraste, la curva de experiencia se refiere a la disminución de los costos que se producen durante el ciclo de vida de un producto, donde los costos a tener en cuenta son los costos totales. En cambio, las curvas de aprendizaje se miden mediante la producción total acumulada hasta la fecha. Por consiguiente, la curva de aprendizaje formula los fenómenos de acomodación o aprendizaje en una progresión geométrica, que expresa el coste decreciente de ejecución de una operación repetitiva cualquiera, a medida que la misma se reitera. Esto ya que, cuando se expide una orden de fabricación de un nuevo producto, el equipo productivo entra en un proceso de adaptación, cuya consecuencia se manifiesta en los tiempos de trabajo requeridos en la elaboración de las primeras unidades de la serie; estos resultan superiores a los tiempos invertidos en la realización de unidades posteriores (Aguirre, 1985).

La formulación estándar de las curvas de aprendizaje se modela como una función de la experiencia acumulativa, en una tarea en particular en lugar de una serie de tareas, por lo que se supone que la tasa de aprendizaje se maximiza mediante la dedicación a una sola actividad. Por ende, se considera que la especialización tiene un impacto en las curvas de aprendizaje (Schilling, Vidal, Ployhart \& Marangoni, 2003).

La importancia del estudio de las curvas de aprendizaje, radica en la necesidad de definir de manera efectiva los planes a seguir en la organización. Huber (1991), dice que la reducción de tiempo y costo en la producción de un nuevo producto, se utiliza con frecuencia en la planificación. Asimismo, Grima y Tena (1984), consideran que al estimarse los parámetros de la curva de aprendizaje, esta se puede emplear para establecer objetivos y planificar. A su vez, Aguirre (1985), expone que la curva de aprendizaje tiene un propósito de control. Además, la curva de aprendizaje ha sido utilizada con éxito por algunas empresas, para estimar costos unitarios de previsión, necesidades de mano de obra y plazos de entrega, cuando se aumenta la producción de una unidad en particular Vásquez, 2017; Lloyd, 1971).

Por su parte, Dolan y Jeuland (1981), tienen en cuenta la tasa de experiencia, como uno de los aspectos a evaluar en la planificación, en la estrategia de precios óptimos de la empresa; además, consideran el efecto de la experiencia en los costos unitarios de producción y comercialización del producto, desde la perspectiva de la curva de experiencia. Igualmente, Amit (1986), plantea la curva de aprendizaje como un factor en la estrategia de liderazgo de costos. Lo anterior permite decir que las curvas de aprendizaje son una herramienta importante en la toma de decisiones para la alta gerencia. Esto debido a que con ellas se puede evaluar el rendimiento obtenido y planificar en base a los efectos del aprendizaje de la organización.

\subsection{Tipos de curvas de aprendizaje}

Las curvas de aprendizaje pueden corresponder tanto al aprendizaje individual como al aprendizaje en grupo u organizacional (Schilling et al., 2003), ver figura 3. A continuación se explican los tipos de curvas de aprendizaje. 


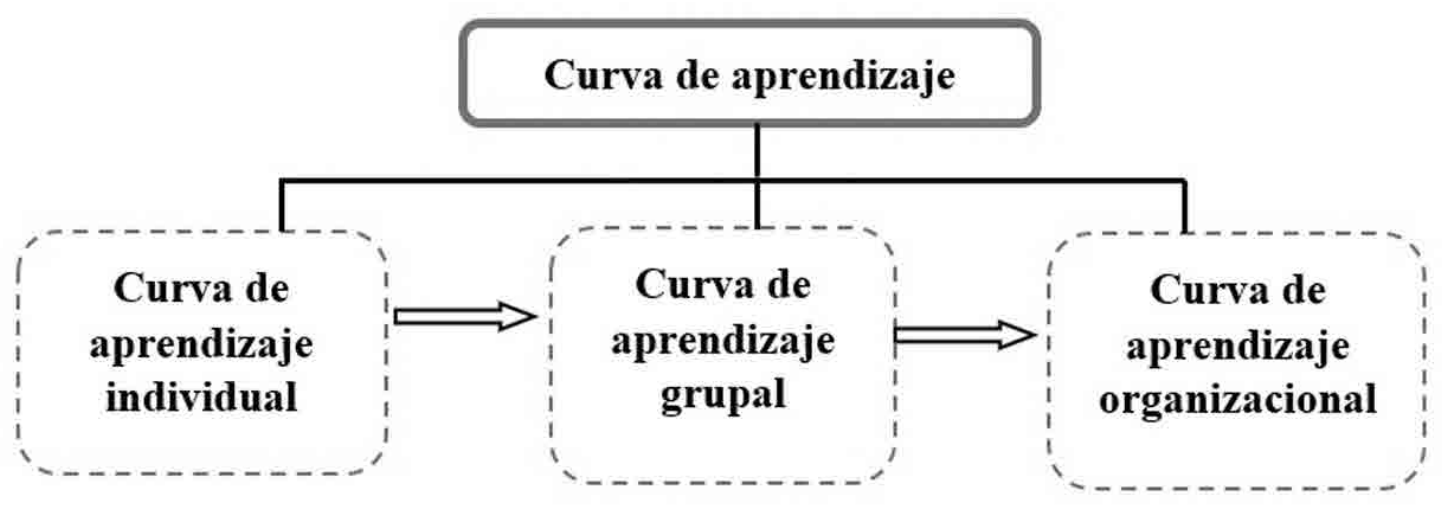

Figura 3. Tipos de curvas de aprendizaje.

\section{Curva de aprendizaje individual}

Este concepto se ha usado en la literatura de la sicología desde finales del siglo XIX y comienzos del XX (Thompson, 2012). Una de las principales demostraciones de curva de aprendizaje individual fue realizada por Ebbinghaus (1885), consistente en memorizar cadenas cada vez más largas de sílabas sin sentido por parte de las personas. Asimismo, William y Harter (1899), realizaron una curva de aprendizaje de telegrafistas durante el envío y recepción de cartas. Los autores observaron que la tasa de progreso dependía en parte de la velocidad de los procesos mentales y nerviosos de cada individuo, pero mucho más de cuánto se incluyó en cada proceso del aprendizaje de la telegrafía.

Por su parte, Ellis (1965), considera la curva de aprendizaje individual como un tipo de transferencia de aprendizaje. Así, dadas las curvas de aprendizaje en una unidad individual de producción, se puede definir el rendimiento general en cualquier unidad, como: la cantidad de rendimiento que se deriva del aprendizaje en las unidades anteriores, que se transfieren a la unidad actual, más un aumento incremental en el rendimiento debido al nuevo aprendizaje adquirido. Además, Glover (1966), hace referencia a las curvas de aprendizaje individual como el proceso de adquisición de habilidades. Es decir, el proceso de transferencia de aprendizaje (learning to learn), indica que los individuos mejoran su aprendizaje a través del tiempo; puesto que transfieren su aprendizaje previo sobre cómo asimilar o procesar determinados tipos de información, para el nuevo conjunto de problemas a resolver (Schilling et al., 2003).

Asimismo, Towill y Bevis (1972), a través de un enfoque de sistemas, construyen un modelo de curva de aprendizaje para derivar un modelo particular del rendimiento de un operario, referente a las tareas repetitivas que realiza. Los autores concluyen que en un sistema de control de gestión, se presenta un aumento de la productividad debido al aprendizaje de tareas repetitivas, por parte de las personas que realizan esa actividad.

Hitchings y Towill (1975), realizan un análisis de los errores del modelo de curvas de aprendizaje de tiempo constante, aplicado al operador humano como un elemento del modelo dinámico del sistema y no como un elemento de la producción. Los autores concluyen que la suma acumulada, muestra cómo pequeños efectos transitorios individualmente insignificantes, se vuelven significativos en un tiempo razonablemente corto, cuando se acumulan los residuos individuales. Además, Bohlen y Barany (1976), modelan curvas de operadores que 
realizan operaciones industriales de aprendizaje, las cuales varían según las características del operador y de la operación que se realiza.

Aunque la curva de aprendizaje también se utiliza para describir el aprendizaje individual, en un proceso industrial el aprendizaje individual no es la causa principal para la reducción de costos. La reducción se da por la combinación de varios factores, como: fuerza de trabajo eficaz, mejor control del proceso de producción, organización más eficaz, introducción de nuevos métodos de producción, nueva tecnología, rediseño del producto, normalización y automatización, entre otros (Moya, Cortés \& Martínez, 2019; Vergara, 2015; Hernández y Sánchez, 2017; Lombana et al., 2017). Algunos de estos factores son dependientes entre sí y no pueden separarse fácilmente (Olsen \& Stordahl, 2004).

\section{Curvas de aprendizaje grupal}

El cálculo de este tipo de curvas, se realiza en grupos que se conforman dentro de una organización. En este caso, se toma la definición de la organización como un grupo de personas organizadas para un propósito particular (Schilling et al., 2003). Estos autores expresan que las organizaciones son típicamente una jerarquía dentro de un sistema de grupos pequeños, y gran parte del aprendizaje se lleva a cabo en la organización desde los niveles de grupos pequeños, por lo que es importante el estudio desde el nivel grupal.

Glock y Jaber (2014), indican que existe diferencia en la forma en que las personas aprenden en grupos y cómo un individuo aprende por sí mismo en un grupo, concluyendo que una curva de aprendizaje organizacional es la agregación de curvas de aprendizaje individuales. Asimismo, Wilson, Goodman y Cronin (2007), consideran que la diferencia en el aprendizaje individual en un grupo y el aprendizaje en grupo, está dada por la transferencia de conocimiento entre los miembros del grupo.

Una aplicación de las curvas de aprendizaje, es la de Towill y Bevis (1972). Ellos no solo calcularon una curva de aprendizaje individual, sino que estimaron un modelo de curva de aprendizaje para grupos de trabajadores. Utilizaron una configuración de diagrama de bloques en un sistema de control de gestión, que mejora la productividad mediante la indicación de las áreas a mejorar en: los programas de formación, estándares de tiempo, patrones de movimiento y sistemas de incentivos. Igualmente, Argote (1993), plantea una curva de aprendizaje para examinar su impacto en el volumen de negocios y tareas complejas, a través de un experimento en grupo. Los resultados evidencian que el desempeño del grupo se ajusta al modelo de curva de aprendizaje a nivel organizacional; además, el rendimiento se afecta negativamente cuando se remplaza un grupo por otro y se aumenta la complejidad de la tarea.

En el estudio de curvas de aprendizaje grupal, Glock y Jaber (2014), proponen un modelo matemático para describir el aprendizaje en grupo en los procesos con y sin rotación de empleados. Los autores concluyen que el aprendizaje grupal es más que una agregación de los procesos de aprendizaje individual. Ellos demuestran que la inserción de un componente relacionado con la transferencia de conocimiento entre los individuos, conduce a un mejor ajuste de la curva de aprendizaje grupal cuando se emplean datos experimentales. Los estudios mencionados anteriormente, evidencian la presencia de aprendizaje individual dentro de un grupo y el aprendizaje en conjunto de un grupo, que se puede estimar a través de la aplicación de modelos de curvas de aprendizaje grupal.

\section{Curvas de aprendizaje organizacional}

En la investigación realizada a la industria de la aviación (Wright, 1936), se evidenció que el costo de producción del armazón de un avión disminuye en la medida que la producción acumulada aumenta. Aunque el autor no define explícitamente el término de curva de aprendizaje organizacional, por primera vez fue estudiada esta relación de manera sistemática, y representada por curvas aplicadas a una industria. Wright (1936), considera que la rela- 
ción entre costo y cantidad acumulada, tiene tres explicaciones: la primera fue, un trabajador mejora sus competencias a través de la práctica; la segunda, está relacionada con la capacidad de usar un mayor número de mano de obra menos calificada, más herramientas y procesos de estandarización; la tercera tiene relación con el mayor uso de maquinaria y mejoras, durante el tiempo de configuración en la producción de grandes cantidades.

Luego del trabajo de Wrigth (1936), se expuso un modelo con el parámetro $b$ negativo, que cambió la estructura del modelo inicial, pero permitió la aplicación de la curva de aprendizaje en otras industrias, como: electrónica, manufactura, industria de fuselaje e industria de capital intensivo. Al igual que en la industria aeronáutica, los estudios indican que el modelo podría ser utilizado para proporcionar una descripción eficiente de la fase de arranque, que se experimenta durante la fabricación de nuevos productos en esas industrias (Baloff, 1966).

La curva de aprendizaje organizacional se especifica en función de la producción acumulada. Ello permite modelar el crecimiento o decrecimiento de los costos contra el número de unidades de producción, por lo general con una tasa decreciente (Schilling et al., 2003; Baloff, 1966; Hatch \& Mowery (1998). A lo largo de este documento, se citan otras investigaciones orientadas al desarrollo de curvas de aprendizaje organizacional, que buscan dar respuesta a las necesidades propias de diversos sectores económicos.

\section{Modelos de curvas de aprendizaje}

\subsection{Modelos clásicos}

\section{Modelos log-lineal}

Entre los modelos que asumen un comportamiento log-lineal, se encuentran los siguientes:

Modelo de Wright o modelo generalizado. Wright (1936), planteó que, si un trabajador realiza la misma tarea varias veces, el tiempo requerido para finalizar esta tarea disminuirá a una velocidad constante. Esto debido a que, los trabajadores aprenden de la experiencia previa y pueden ser más eficientes en la realización de la misma. Además, identificó en la producción de aviones, una tasa de aprendizaje de $80 \%$, o una mejora del $20 \%$ de la producción, debido a que el número de unidades de producción se duplicó.

Modelo de Crawford. Crawford (1944), usa la fórmula del modelo de Wright, considerando que es una función exponencial donde la disminución del tiempo de producción es una función del número de unidades producidas. Además, estima los tiempos individuales (o costos relacionados) para producir una determinada unidad. El parámetro en el modelo es el coeficiente de la curva de aprendizaje K, el cual indica la reducción de tiempo de producción cuando el volumen de producción se duplica. K es un número menor que uno, usualmente expresado en porcentaje que oscila entre $70 \%$ y $90 \%$.

Por su parte, Olsen y Stordahl (2004), plantean que la principal diferencia entre los modelos log-Lineal de Wright y Crawford, es que el primero describe el efecto acumulativo del aprendizaje, mientras que el segundo se refiere solo a una escala de efectos.

\section{ModeloS}

Carr (1946), consideró que había un error en el modelo de Wright, pues asumió un aprendizaje constante. Por ende, su modelo de curva S, supone un aumento gradual del aprendizaje en las primeras etapas de la producción, seguido por un período de máximo rendimiento. Esta acumulación de aprendizaje se suele atribuir a: las personas, los cambios en el procedimiento y el tiempo necesario para aprender el manejo de una nueva máquina. Estos ajustes ocurren temprano (primera etapa), en el proceso de producción (Moore et al., 2015). 


\section{Modelo DeJong}

El modelo DeJong también es una derivación del modelo de Wright. Sin embargo, incluye un factor de incomprensibilidad, denotado por la letra $\mathrm{M}$, el cual representa la relación entre los procesos manuales realizados por los operarios y los procesos de la máquina (Carlson, 1964). El factor de incomprensibilidad es una constante entre cero y uno. Si toma el valor cero implica un proceso de operación totalmente manual y si toma el valor uno, indica una operación dominada completamente por una máquina (Badiru, Elshaw \& Everly, 2013).

\section{Modelo Stanford- $B$}

Este modelo, desarrollado por Yelle (1979), introduce el parámetro equivalente a la unidad de experiencia de la ecuación propuesta en el modelo de Wright. Este parámetro, representado por B, es una constante de cero a diez, que representa el número de unidades producidas antes del inicio de la producción de la primera unidad, y es la pendiente asíntota de la curva de aprendizaje. Si este factor es cero, el modelo se estima como el modelo original de Wright (Badiru, 2012). Por el contrario, si el factor es diez, los efectos del aprendizaje comenzarán en la unidad 11, y la disminución en el rendimiento se producirá en un menor tiempo, haciendo que la curva de aprendizaje se aplane rápidamente (Moore et al., 2015).

3.2 Extensiones de los modelos de curvas de aprendizaje

El modelo de curva de aprendizaje creado por Wright, en el transcurso de los años ha presentado transformaciones como las indicadas anteriormente. Sin embargo, existen otras variaciones en su formulación matemática, lo que impide hablar de un modelo único, debido a las aplicaciones realizadas en industrias diferentes a la aviación (Baloff, 1966). A continuación se explican algunas de las modificaciones realizadas.
Baloff (1966), considera que el modelo de Wright no es totalmente compatible con aplicaciones en el uso de maquinaria intensiva en manufactura. Por ello utiliza una curva de aprendizaje con el parámetro $b$ negativo, aplicada a varias industrias de capital intensivo, que también es compatible con la mayoría de aplicaciones en manufactura con mano de obra intensiva. Sin embargo, las diferencias entre los modelos no parecen afectar su eficacia en la descripción de los aumentos de productividad, experimentados durante la fase inicial de la operación.

Asimismo, Bevis, Finnear y Towill (1970), propusieron un modelo de curva de aprendizaje y desarrollaron un logaritmo para estimar los parámetros tempranos durante el proceso de aprendizaje. Towill y Bevis (1972), aplicaron el modelo para estimar costos, comparar esquemas de formación y seleccionar operaciones para mejorar la utilización de los recursos en la consecución de los fines de la organización. Además, Towill (1973), usa ese modelo como referencia para estimar los parámetros de tiempo constante del modelo de curva de aprendizaje, a partir de datos históricos.

En el modelo de Hitchings y Towill (1975), se asume la dispersión de las observaciones en la práctica, desarrollando una ecuación que incluye el término de error. Los autores emplean el criterio de bondad de ajuste, referente a la suma mínima de los errores al cuadrado, para obtener un mejor ajuste de la curva de aprendizaje. En este caso, el valor de T puede ser considerado como una medida de la complejidad del trabajo y se espera una mejora significativa en el rendimiento, incluso cuando el tiempo transcurrido son varios múltiplos de T. La existencia de estabilidad en la complejidad del trabajo, se considera un medio para evaluar la eficacia o el método de entrenamiento.

Bohlen y Barany (1976), desarrollaron un método para predecir la curva de aprendizaje de un operador que realiza una operación específica de mon- 
taje, basado en ciertas mediciones del operador y de la operación. Los autores utilizaron una función lineal, porque las curvas de aprendizaje modelan los valores reales del rendimiento de aprendizaje. Otra ventaja de esta función, es la de tener una asíntota a un valor finito, que representa la salida máxima del trabajador.

Por su parte, Spence (1981), desarrolla un modelo de interacción competitiva en una industria, donde el costo unitario se reduce con la producción. Esto debido a que la curva de aprendizaje es una función que relaciona los costos unitarios de la empresa con el volumen acumulado. Además, utilizó un modelo de dos periodos para explorar las diferencias entre los equilibrios de apertura y cierre, para evaluar el impacto del aprendizaje en los efectos indirectos de una empresa a la otra.

Desde otra perspectiva, Mazzola y McCardle (1996), plantean un modelo de decisión de producción óptima en presencia de una curva de aprendizaje incierta, donde algunos de los parámetros de la función de costo son variables aleatorias. Ellos consideran que los costos son exponencialmente decrecientes en la producción acumulada, con alguna variación aleatoria en los costos observados. Los autores asumen en el modelo de horizonte infinito, que los parámetros de la distribución son desconocidos, aplicando el Teorema de Bayes a una distribución de probabilidad a priori. Este modelo permitió probar que una función única de valor acotado, satisface la recurrencia estocástica dinámica y la existencia de una política óptima. Además, se plantea un modelo finito cuando existe un número finito de periodos, calculando una política óptima en este escenario.

Un desafío que enfrentan las empresas, es lograr mejoras continuas en sus procesos para garantizar la calidad de los productos (Hernández, Figueroa \& Correa, 2018; Sáenz, Lugo \& Zapata, 2019). En este sentido, Zangwill y Kantor (1998), proponen un modelo para determinar la cantidad de aprendizaje que se da en una mejora de procesos incluyendo los costos directos, como alternativa al método usado comúnmente donde se incluyen los costos totales. El método directo reduce sustancialmente los errores del método anterior, obteniendo mejoras en los valores calculados tanto en la curva de aprendizaje como de mejora continua. Los autores formulan una ecuación diferencial que no sólo caracteriza la mejora continua, sino también revela cómo podría producirse el aprendizaje a través de la curva. Lo anterior con el fin de ayudar a la gerencia de una empresa a evaluar la eficacia de los distintos procedimientos, permitiéndole plantear mejoras a los procesos que llevan a cabo.

Otro aspecto a considerar en una organización es la productividad. Thomas, Mathews y Ward (1986), desarrollan una investigación aplicada a la productividad en la construcción, determinando la correlación entre los precios unitarios previstos y los reales. Sobre esta base modelaron las tarifas unitarias para 65 conjuntos de datos, con cinco modelos diferentes. Los autores concluyen que el modelo de curva de aprendizaje cúbico, es el mejor predictor de los rendimientos futuros, pues la tasa de aprendizaje no es un valor constante. En contraste, Everett y Farghal (1994), exponen que el modelo de curva de aprendizaje cúbico no es un buen predictor de los rendimientos futuros. Sin embargo, destacan que al determinar la relación entre el tiempo de actividad o costo y el número de ciclo, este modelo proporciona un ajuste estadístico fiable y una alta correlación con actividades repetitivas completas.

En esta misma línea, Hinze y Olbina (2009), llevaron a cabo un análisis empírico aplicado a la fabricación de pilotes, para determinar si las curvas de aprendizaje podrían predecir con precisión los esfuerzos de producción de unidades futuras. Los resultados indican que el equipo de fabricación de los pilotes, presenta una ligera mejoría en el aprendizaje a lo largo del tiempo empleado en este esfuerzo. Así mismo, las predicciones sobre la cantidad de esfuerzo para fabricar pilotes con curva de aprendizaje, son razonablemente precisas; lo que demuestra que este modelo se adecúa a grandes cantidades de elementos repetidos. 
Jarkas (2010), en una investigación sobre la fijación de los insumos de trabajo de vigas y losas, en 21 edificios residenciales de varios pisos, utilizaron el método de Mínimos Cuadrados Ordinarios (MCO). Los resultados indican que la mayoría de las casas observadas, presentan un aumento o una reducción insignificante en el trabajo. Esto es contrario a la teoría de la curva de aprendizaje, donde se espera una disminución de los insumos de trabajo (mano de obra), en la medida que aumenta el número de ciclos. Asimismo, Jarkas y Horner (2011), corroboran lo anterior realizando una aplicación similar en el sector de la construcción.

En el trabajo de Arias (2014), se realiza la modelación de curvas de aprendizaje para Tecnologías de Energía Renovable en Colombia, estimando la curva por MCO. Los resultados indican que los costos de infraestructura y costos totales se ven afectados por economías de escala, mientras que el costo de los equipos se determina por el proceso de learning by doing. En este caso, la tasa de aprendizaje oscila entre 20 y $24 \%$. El autor concluye que si se excluyen los efectos del learning by doing, se puede subestimar la rentabilidad de la tecnología.

Lo anterior indica que las curvas de aprendizaje, si bien iniciaron en la industria de la aviación, se han difundido a diversos sectores industriales, con la aportación de nuevos modelos matemáticos para su estimación (Panas \& Pantouvakis, 2018). Los trabajos revisados destacan el beneficio de las curvas de aprendizaje en temas como: la planeación, la capacitación y el control de procesos. Igualmente, los resultados de la aplicación de las curvas de aprendizaje en el sector de la construcción, generan interrogantes sobre su eficiencia en este sector.

\section{Conclusiones}

La revisión realizada indica la importancia del estudio de las curvas de aprendizaje, tanto a nivel individual, grupal y organizacional. A nivel individual, se resalta que la curva de aprendizaje se manifiesta en la transferencia de conocimiento entre los colaboradores de una empresa. A su vez, desde el punto de vista de la curva de aprendizaje en grupo, se evidencian tanto el aprendizaje individual en un grupo, así como el aprendizaje en conjunto de un grupo. Igualmente, las curvas de aprendizaje organizacional pueden ser fuente para la toma de decisión en las empresas. Esto debido a que los distintos modelos de curva de aprendizaje, pueden obtener información referente a: metas de producción, evaluación de rendimientos, estimación de mano de obra, optimización de precios y liderazgo de costos, para crear ventajas competitivas y tasas de aprendizaje.

En cuanto a los modelos de curvas de aprendizaje y su extensión, se observa la eficiencia de la aplicación de los mismos, en diversos sectores industriales. Con ellos se logra obtener beneficios como los indicados anteriormente, aunque los autores en algunos planteamientos difieran en la aplicación de los modelos. Sin embargo, en los estudios consultados sobre el sector de la construcción, la eficiencia de los modelos no es clara.

En este sentido, queda el debate respecto al sector de la construcción, así como la posibilidad de realizar aplicaciones de las curvas de aprendizaje en futuros estudios. Asimismo, sería importante desarrollar investigaciones de curvas de aprendizaje aplicadas al sector servicios. Esto permitiría contrastar la eficiencia de estos modelos, logrando mayores aportes al tema de las curvas de aprendizaje, desde la perspectiva individual, grupal u organizacional.

A parte de lo anterior, se deben llevar a cabo estudios que profundicen en la inclusión del ausentismo como un factor relevante en las curvas de aprendizaje. Esto debido a que puede ser visto como una consecuencia de que los colaboradores de una empresa no se involucren en el aprendizaje. Además, sería interesante realizar estudios para determinar si las pruebas de selección de personal, deberían estar en función de las curvas de aprendizaje de la organización. 


\section{Referencias}

Aguirre-Sádaba, A. A. (1985). Las curvas de aprendizaje y sus aplicaciones. Cuadernos de Ciencias Económicas Y Empresariales, 16, 121-134. Recuperado de: http://cuadernos.uma.es/pdfs/pdf318. pdf

Alean-Pico, A., Del Rio, J., Simancas-Trujillo, R., \& Rodríguez-Arias, C. (2017). ¿El emprendimiento como estrategia para el desarrollo humano y social?. Saber, Ciencia Y Libertad, 12 (1), 107-123. Recuperado de: http://www.sabercienciaylibertad. org/ojs/index.php/scyl/article/view/218

Amit, R. (1986). Cost leadership strategy and experience curves. Strategic Management Journal, 7 (3), 281-292. doi: http://doi.org/10.1002/ smj.4250070308

Arias-Gaviria, J. (2014). Modelamiento y Simulación de Curvas de Aprendizaje para Tecnologías de Energía Renovable en Colombia (Tesis de maestría). Medellín, Colombia: Universidad Nacional de Colombia. Recuperado de: bdigital.unal.edu. co/43657/1/1020439952.2014.pdf

Argyris, C., \& Schon, D. (1978). Organizational Learning. Reading. MA: Addison-Wesley.

Argote, L. (1993). Group and organizational learning curves: individual system and environmental comoponents. British Journal of Social Psychology, $32(1), 31-51$.

Arrow, K. J. (1962). The Economic Implications of Learning by Doing. The Review of Economic Studies, 29 (3), 155-173.

Badiru, A. (2012). Half-life learning curves in the defense acquisition life cycle. Defense ARJ, 19 (3), 283-308. Recuperado de: https://www.afit.edu/ BIOS/publications/HalflifeLearningCurvesinDefenseAcquisitionLifeCycleBadiruDARJ2012.pdf
Badiru, A., Elshaw, J., \& Everly, M. (2013). Half-life learning curve computations for airframe lyfe -cycle costing of composite manufacturing. Journal of Aviation and Aerospace Perspectives, 3 (2), 6-37.

Baloff, N. (1966). the learning curve-some controversial issues. The Journal of Industrial Economics, 14 (3), 275-282.

Panas, A., \& Pantouvakis, J. (2018). On the use of learning curves for the estimation of construction productivity. International Journal of Construction Management, 18 (4), 301-309. doi: 10.1080/15623599.2017.1326302

Bevis, F. W., Finnear, C., \& Towill, D. R. (1970). Prediction of operator performance during learning of repetitive tasks. International Journal of Production Research, 8, 293.

Bohlen, G. A., \& Barany, J. W. (1976). A learning curve prediction model for operators performing industrial bench assembly operations. International Journal of Production Research, 95-303.

Cangelosi, V., \& Dill, W. (1965). Organizational Learning: Observations Toward a Theory. Administrative Science Quarterly, 10 (2), 175-203. doi: http:// doi.org/10.2307/2391412

Carlson, J. G. (1964). Normes de temps pour la fabrication en petite serie. (B. T. E. L'Etude du Travail, Ed.). París, Francia.

Carr, G. W. (1946). Peacetime cost estimating requires new learning curves. Aviation, 45, 76-77.

Cook, S. D. N., \& Yanow, D. (1993). Culture and Organizational Learning. Journal of Management Inquiry, 2 (4), 373-390. doi: http://doi. org/10.1177/105649269324010

Crawford, J. R. (1944). Learning Curve, ship curve, ratios, related data. Ockheed Aircraft Corporation. 
Crossan, M. M., Lane, H. W., White, R. E., \& White, E. (1999). Learning From Intuition To Framework. Academy of Management Review, 24 (3), 522537. Recuperado de: http://www.jstor.org/stable/10.2307/259140

Del Río-Cortina, J., Cardona -Arbeláez, D., \& Guacarí-Villalba, A. (2017). Responsabilidad social empresarial y construcción de la marca: una nueva mirada a las estrategias de gestión. Revista de Investigación, Desarrollo e Innovación, 8 (1), 49-60. doi: https://doi.org/10.19053/20278306. v8.n1.2017.7370

Dolan, R. J., \& Jeuland, A. P. (1981). Experience Curves and Dynamic Demand Models: Implications for Optimal Pricing Strategies. Journal of Marketing, 45 (1), 52. http://doi.org/10.2307/1251720

Dutton, J. M., Thomas, A., \& Butles, J. E. (1984). The history of progress functions as a managerial technology. Bus. Hist. Rev., 58, 204-233.

Ellis, H. C. (1965). The transfer of learning. MacmiIlan Company. Oxford, England.

Ebbinghaus, H. (1885). Memory: A contribution to experimental psychology, 20 (4), 155-157.

Everett, J. G., \& Farghal, S. (1994). Learning Curve Predictors for Construction Field Operations. Journal of Construction Engineering and Management, 120 (3), 603-616. doi: http://ascelibrary.org/doi/ abs/10.1061/(ASCE)0733-9364(1994)120:3(603)

Fernández-Guerrero, M. M., \& González-Ferro, V. (2017). Enseñar, el arte de transformar y crecer. Saber, Ciencia y Libertad, 12 (2), 167-174. Recuperado de: http://www.sabercienciaylibertad.org/ojs/index.php/scyl/article/view/242

Fiol, M., \& Lyles, M. (1985). Organizational learning. The Academy of Management Review, 10 (4), 803813. doi: http://doi.org/10.2307/258048
Glock, C., \& Jaber, M. (2014). A group learning curve model with and without worker turnover. Journal of Modelling in Management, 9 (2), 179-199. doi: http://doi.org/10.1108/JM2-05-2013-0018

Glover, J. H. (1966). Manufacturing progress functions: an alternative model and its comparison with existing functions. International Journal of Production Research, 4, 279.

Grima-Terre, J. D., \& Tena-Millan, J. (1984). Análisis y formulación de la estrategia empresarial. Barcelona: Ed. Hispano Europea S.A.

Hatch, N. W., \& Mowery, D. C. (1998). Process Innovation and Learning by Doing in Semiconductor Manufacturing. Management Science, 44 (11), 1461-1477.

Hall, G., \& Howell, S. (1985). The Experience Curve from the Economist's Perspective. Strategic. Management Journal, 6 (3), 197-212.

Hernández-Fuentes, S., \& Sánchez-Mojica, K. (2017). Innovación y competitividad: micro y pequeñas empresas del sector agroindustrial en Cúcuta. Revista de Investigación, Desarrollo e Innovación, 8 (1), 23-33. doi: https://doi.org/10.19053/20278306. v8.n1.2017.7368

Hernández-Gil, C., Figueroa-Ramírez, E. F., \& Correa-Corrales, L. E. (2018). Reposicionamiento de marca: el camino hacia la competitividad de las pequeñas y medianas empresas. Revista de Investigación, Desarrollo e Innovación, 9 (1), 33-46. doi: https://doi.org/10.19053/20278306.v9.n1.2018.8505

Hitchings, B., \& Towill, D. (1975). An error analysis of the time constant learning curve model. International Journal of Production Research, 13 (2), 105-135.

Hinze, J., \& Olbina, S. (2009). Empirical Analysis of the Learning Curve Principle in Prestressed Concrete Piles. Journal of Construction Enginee- 
ring and Management, 425-431. Recuperado de: https://ascelibrary.org/doi/10.1061/\%28ASCE\%29C0.1943-7862.0000004

Huber, G. P. (1991). Organizational Learning: The Contributing Processes and the Literatures. Organization Science, 2 (1), 88-115. doi: http://doi. org/10.1287/orsc.2.1.88

Hult, G. T. M., Ketchen, D. J., \& Reus, T. H. (2001). Organizational learning capacity and internal customer orientation within strategic sourcing units. Journal of Quality Management, 6 (2), 173-192.

Jarkas, A. M. (2010). Critical Investigation into the Applicability of the Learning Curve Theory to Rebar Fixing Labor Productivity. Journal of Construction Engineering and Management, 136 (12), 1279-1288. doi: http://doi.org/10.1061/(ASCE) C0.1943-7862.0000236

Jarkas, A., \& Horner, M. (2011). Revisiting the applicability of learning curve theory to formwork labour productivity. Construction Management and Economics, 29 (5), 483-493. doi: http://doi.org/10. 1080/01446193.2011.562911

Kim, D. H. (1993). The link between individual and organizational learning. MIT Sloan Management Review, 35 (1), 37-50.

Levitt, B., \& March, J. (1988). Organizational Learning. Annual Review of Sociology, 319340. doi: http://doi.org/Doi 10.1146/Annurev. So.14.080188.001535

Lloyd, N. (1971). Productivity Gains with the learning curve. Industrial Management, 9-12.

Lombana, J., Amashta, Y., Correa, C., \& Rodríguez, M. C. (2017). Benchmarking y análisis de competitividad de las cadenas productivas de quinua en Colombia, Perú y Bolivia. FACE: Revista de la Facultad de Ciencias Económicas y Empresariales, 17 (2), 157-163. Recuperado de: http://revistas.unipam-
plona.edu.co/ojs_viceinves/index.php/FACE/article/view/2891

Mazzola, J. B., \& McCardle, K. F. (1996). A Bayesian Approach to Managing Learning-Curve Uncertainty. Management Accounting, 42 (5), 680-692.

Moore, J. R., Elshaw, J. J., Badiru, A. B., \& Ritschel, J. D. (2015). Acquisition challenge: the importance of incompressibility in Comparing Learning Curve Models. Publication of the Defense Acquisition University, 22 (4), 416-449. Recuperado de: https://pdfs.semanticscholar.org/21a4/dd13cb9075f01f02a3d1a81b1128bdbf1bf9.pdf

Moya-Espinosa, P. I., Cortés-Rodríguez, N. C., \& Martínez-Cárdenas, A. G. (2019). Proceso de toma de decisiones en mipymes hoteleras de Boyacá, Colombia. Revista de Investigación, Desarrollo e Innovación, 9 (2), 237-248. doi: 10.19053/20278306. v9.n2.2019.9159

Olsen, B. T., \& Stordahl, K. (2004). Models for forecasting cost evolution of components and technologies. Telektronikk, 4, 138-144.

Ordóñez-Ortega, O., Gualdrón-Pinto, E., \& Amaya-Franky, G. (2019). Pensamiento variacional mediado con baldosas algebraicas y manipuladores virtuales. Revista de Investigación, Desarrollo e Innovación, 9 (2), 347-362. doi: 10.19053/20278306. v9.n2.2019.9180

Sáenz-Zapata, J., Lugo-Arias, E., \& Parada-Pérez, J. (2019). Determinantes de la competitividad internacional del Departamento del Magdalena Colombia: Un enfoque desde la Teoría de la Complejidad Económica. Saber, Ciencia Y Libertad, 14 (1), 190-210. doi: https://doi.org/10.18041/23823240/saber.2019v14n1.5235

Schilling, M. a., Vidal, P., Ployhart, R. E., \& Marangoni, A. (2003). Learning by Doing Something Else: Variation, Relatedness, and the Learning Curve. 
Management Science, 49 (1), 39-56. doi: http://doi. org/10.1287/mnsc.49.1.39.12750

Simon, H. a. (1991). Bounded Rationality and Organizational Learning. Organization Science, 2 (1), 125-134. doi: http://doi.org/10.1287/orsc.2.1.125

Sinkula, J. M. (1994). Market information processing and organizational learning. The Journal of Marketing, 35-45.

Spence, M. (1981). The Learning Curve and competition. The Bell Journal of Economics, 12 (1), 49-70. doi: http://doi.org/DOI: 10.2307/3003508

Thomas, H. R., Mathews, C. T., \& Ward, J. G. (1986). Learning Curve Models of Construction Productivity. Journal of Construction Engineering and Management, 112 (2), 245-258. doi: https://ascelibrary. org/doi/10.1061/\%28ASCE\%290733-9364\%28198 6\%29112\%3A2\%28245\%29

Thompson, P. (2012). for Organizational Learningby-Doing, 26 (3), 203-224.

Towill, D. R. (1973). A direct method the determination of learning curve parameters from historical data. International Journal of Production Research, 11 (1). Recuperado de: https://www.tandfonline. com/doi/abs/10.1080/00207547308929949

Towill, D. R., \& Bevis, F. W. (1972). Manegeral control system based on Learning Curve models. International Journal of Production Research, 11 (3), 219-238. Recuperado de: https://www.tandfonline.com/doi/abs/10.1080/00207547308929967

Ulrich, D., Von Glinow, M., Jick, T., Yeung, A., \& Nason, S. (1994). Learning organization, culture chan- ge, and competitiveness: How managers can build learning capability. Lexington, MA: The International Consolidation for Executive Development Research.

Vásquez-Roldán, J. S. (2017). El papel del capital humano y las mediciones alternativas de la productividad en la dinámica industrial y los mundos de producción. Revista Espacios, 38 (57), 15. Recuperado de: https://www.revistaespacios.com/ a17v38n57/17385715.html

Vergara-Arrieta, J. J. (2015). Los sistemas de medición del desempeño estratégico frente a la competitividad y sustentabilidad. Saber, Ciencia y Libertad, 10 (2), 17-26. Recuperado de: https://dialnet. unirioja.es/servlet/articulo?codigo $=5295019$

William, L. B., \& Harter, N. (1899). Studies on the telegraphic language: The acquisition of a hierarchy of habits. Psychological Review, 6, 345-375.

Wilson, J. M., Goodman, P. S., \& Cronin, M. A. (2007). Group Organizational. Academy of Management Review, 32 (4), 1041-1059.

Wright, T. P. (1936). Factors Effecting the Cost of Airplanes. Journal of Aereonautical Sciences, 3 (4), 122-128.

Yelle, L. E. (1979). He learning curve: historical review and comprehensive study. Decision Science, $10(2), 302-328$.

Zangwill, W. I., \& Kantor, P. B. (1998). Toward a Theory of Continuous Improvement and the Learning Curve. Management Science, 44 (7), 910-920. http://doi.org/10.1287/mnsc.44.7.910 
